\title{
Multiagent imaging: from the pathophysiologic basis to clinical characterization and radionuclide therapy
}

\author{
Giuliano Mariani · H. William Strauss
}

Received: 5 December 2013/Accepted: 10 December 2013/Published online: 19 December 2013

(C) Italian Association of Nuclear Medicine and Molecular Imaging 2013

The remarkable growth of nuclear medicine in the past 20 years is attributable to a number of factors, including the clinical recognition that imaging with $\left[{ }^{18} \mathrm{~F}\right] \mathrm{FDG}$, touted as the "molecule of the millennium" [1], improves diagnosis and staging, especially when it is used with devices that provide anatomic reference for the metabolic images. Furthermore, the growth of FDG PET/CT has been paralleled by an evolution of other applications of "molecular imaging" for both diagnostic and therapeutic purposes [1].

This issue of Clinical and Translational Imaging focuses on the imaging of multiple parameters (achieved either by imaging with multiple agents or by imaging at multiple time points) as a means of better characterizing the presence or severity of a disorder. In the opening article, $\mathrm{Ci}$ armiello et al. [2] provide a comprehensive review of the complex pathophysiology of degenerative brain disorders. In this regard, both PET and SPECT imaging use a wide range of tracers to track pathophysiological processes in the healthy and diseased brain, especially for the purpose of investigating the pathophysiological aspects of neurodegenerative disorders. This area of investigation is extremely important considering the aging of the global population and the high prevalence of brain disorders, such as Alzheimer's disease and Parkinson's disease, in elderly persons. Utilizing molecular imaging in routine clinical practice may overcome the limitations of a diagnostic approach based solely on clinical judgment and structural

\section{G. Mariani $(\bowtie)$}

Regional Center of Nuclear Medicine, University of Pisa,

Via Roma 67, 56126 Pisa, Italy

e-mail: giuliano.mariani@med.unipi.it

H. W. Strauss

Molecular Imaging and Therapy Service, Memorial Sloan Kettering Cancer Center, New York, NY, USA imaging. These molecular imaging techniques will foster the development of new drugs whose effects on specific pathways can be monitored, and compared to clinical outcomes.

Strauss and Fox explore new techniques that expand the applications of approved radiopharmaceuticals in patients with heart failure and atherosclerosis [3]. Their article focuses mainly on the evaluation of mitochondrial function and sympathetic innervation in patients with heart failure. The new applications they discuss, by providing functional information with high predictive value, allow identification of heart failure patients at high risk of clinical events. Another area of growing interest is linked to the possibility of directly imaging and evaluating focal vascular inflammation, microcalcification and calcification in patients with dyslipidemia. Imaging-based characterization of inflammation, or early phases of calcification in atheromas, is expected to stimulate the development of therapeutic strategies that are more personalized than those based on traditional measurements of lipid levels in patients with dyslipidemia. Characterization of atheromas, especially in the coronary and carotid arteries, may be the imaging approach of choice to permit effective pharmacologic therapy geared at reducing the incidence and severity of clinical events.

Palestro et al. [4] discuss the current role of molecular imaging with single-photon and positron-emitting tracers in the evaluation of inflammation and infection. After reviewing the clinical role of well-established imaging procedures based on approved radiopharmaceuticals (chiefly, labeled autologous leukocytes and $\left[{ }^{18} \mathrm{~F}\right] \mathrm{FDG}$, even though there is still a role for ${ }^{67} \mathrm{Ga}$-citrate scintigraphy), the authors describe ongoing investigations of tracers, such as radiolabeled antibiotics - these were originally expected to be "infection-specific" imaging agents, but to 
date have given somewhat disappointing results-and radiolabeled antimicrobial peptides, which hold promise as infection-specific tracers. Furthermore, with the new tracers being used as objective biomarkers for assessing response to therapy, the role of radionuclide imaging of infection/inflammation seems to be shifting away from diagnosis. These tracers can also potentially be used to target cells and molecules with specific receptor expression for histological characterization, with a view to selecting patients for receptor-targeted therapy as well as for predicting response to treatment.

The remaining manuscripts deal with the characterization and treatment of tumors. The first of these reviews, by Larson et al. [5], focuses on the complex and multifaceted (yet fascinating) biology of neoplastic transformation, as a basis for developing new targeted approaches, using radionuclides, for cancer diagnosis and therapy. This article looks at the biologic events and aspects relevant to tumor mass imaging, in particular tumor-induced neo-angiogenesis, tumor hypoxia and cellular constituents. The authors discuss the concept of immunologic sanctuary of tumors and describe recent therapeutic advances that may help to overcome this biologic problem. This approach is expected to expand the opportunities for molecular targeting with radionuclides in patients with cancer.

Several biologic peculiarities characterize a complex family of tumors traditionally referred to as "neuroendocrine neoplasms". Volterrani et al. [6] review the state of the art of radionuclide targeting of these tumors that, while generally rare, offer great potential for the development of targeted, tumor-specific approaches that could constitute a model for other cancers. Most of these approaches are based on enhanced expression of receptors for somatostatin on the cell surface, or on specific metabolic pathways that are enhanced in certain types of neuroendocrine neoplasms. Approaches based on these biologic characteristics are shifting from the field of single-photon to that of PET imaging, and the identification of new radiopharmaceuticals specific for different targets constitutes the basis not only of new diagnostic imaging approaches, but also of new therapeutic applications.

The innovative strategy of intra-arterial radioembolization (RE) using microspheres labeled with the high-energy beta-emitter ${ }^{90} \mathrm{Y}$ for primary and secondary hepatic malignancies is attracting growing attention as a beneficial treatment for patients who have basically failed several lines of therapy. As reviewed by Maccauro et al. [7], multiagent targeting is, in such cases, a fundamental requisite for the success of the therapeutic procedure. In particular, after evaluating all the clinical parameters that patients have to meet to be considered as candidates for such therapy, hepatic perfusion imaging with ${ }^{99 \mathrm{~m}} \mathrm{Tc}-\mathrm{MAA}$ is performed prior to RE, in order to identify possible extra-hepatic accumulation of radiopharmaceutical and lung shunt, which may make the patient unsuitable for this type of therapy. Furthermore, important recent developments have underlined the role of ${ }^{99 \mathrm{~m}} \mathrm{Tc}$ MAA SPECT-based dose planning in the optimization of RE efficacy.

In the final article, KleinJan et al. discuss the growing role of radioguided surgery, looking at radioguidance as a standalone technique and as a technique used in combination with intraoperative optical guidance. Radioguided surgery can be achieved using multiple agents or even "hybrid" agents, i.e., a radiocolloid that is also tagged with a fluorescent moiety for simultaneous intraoperative detection of radioactive counts and of the fluorescent signal for optical guidance [8]. Furthermore, new radioactive agents will soon be available that localize in the sentinel lymph nodes not because of phagocytosis of the radiocolloid by macrophages, but because of receptor-mediated interaction with macrophages. Also, these agents can be doubly labeled, i.e., simultaneously with a radionuclide (typically ${ }^{99 \mathrm{~m}} \mathrm{Tc}$ ) and a fluorescent moiety. Crucial to application of these latest strategies for intraoperative guidance is the parallel development of new probes with the ability to simultaneously detect radioactivity and the optical signal.

Conflict of interest The authors G. Mariani and H. W. Strauss declare that there is no conflict of interest in relation to this article.

Human and Animal Studies This article does not contain any studies with human or animal subjects performed by any of the authors.

\section{References}

1. Britz-Cunningham SH, Adelstein SJ (2003) Molecular targeting with radionuclides: state of the science. J Nucl Med 44:1945-1961

2. Ciarmiello A, Gaeta C, Guidotti C, del Sette M (2013) Multiagent imaging of the brain. Clin Transl Imaging. doi:10.1007/s40336013-0042-y

3. Strauss HW, Fox J (2013) Additional applications of approved radiopharmaceuticals for nuclear cardiology. Clin Transl Imaging. doi:10.1007/s40336-013-0038-7

4. Palestro CJ, Glaudemans AWJM, Diercks RAJO (2013) Multiagent imaging of inflammation and infection with radionuclides. Clin Transl Imaging. doi:10.1007/s40336-013-0041-z

5. Larson SM, Mariani G, Strauss HW (2013) Tumor biology as a basis for molecular targeting in cancer. Clin Transl Imaging. doi:10.1007/s40336-013-0044-9

6. Volterrani D, Orsini F, Chiacchio S, Bodei L (2013) Multiagent targeting of neuroendocrine neoplasms. Clin Transl Imaging. doi:10.1007/s40336-013-0043-x

7. Maccauro M, Lorenzoni A, Boni G, Chiesa C, Spreafico C, Romito R, Mazzaferro V, Seregni E (2013) Multiagent imaging of liver tumors with reference to intra-arterial radioembolization. Clin Transl Imaging. doi:10.1007/s40336-013-0040-0

8. KleinJan GH, Bunschoten A, Brouwer OR, van den Berg NS, Valdés-Olmos RA, van Leeuwen FWB (2013) Multimodal imaging in radioguided surgery. Clin Transl Imaging. doi: 10.1007/ s40336-013-0039-6 